\title{
Fluorescein angiography and fluorophotometry of the iris in pseudoexfoliation of the lens capsule
}

\author{
ANNE M. V. BROOKS AND W. E. GILliES \\ From the Glaucoma Clinic, Royal Victorian Eye and Ear Hospital, Melbourne, Australia
}

SUMMARY Fluorescein iris angiography and fluorophotometry were performed on a series of 9 patients with bilateral and 11 with unilateral pseudoexfoliation, 12 bilateral aphakes with pseudoexfoliation, and 7 unilateral aphakes with bilateral pseudoexfoliation. Angiography showed a loss of radial iris vessels, a heavy leak of fluorescein from the pupil margin, progressive neovascularisation of the outer $2 / 3$ of the iris, and less constantly a network of fine new vessels in the inner $1 / 3$ of the iris stroma. These changes were absent in unaffected eyes. After cataract extraction there seemed to be a definite lessening of fluorescein leak from the pupil margin. Fluorophotometry showed a much higher fluorescein concentration at the anterior focus in eyes with pseudoexfoliation than in normal controls or in fellow unaffected eyes. There was a much smaller rise in fluorescein concentration at the posterior focus in a minority of affected eyes. The ranges of fluorescein concentrations at the anterior focus in both phakic and aphakic patients with bilateral pseudoexfoliation did not differ significantly. The concentration at the anterior focus of unilateral aphakes with bilateral pseudoexfoliation was lower than in the fellow phakic eye. These findings suggest that the neovascular reaction seen in pseudoexfoliation is associated with patchy occlusion of the normal iris vasculature, occurs in the anterior segment of the eye, and does not continue to progress after removal of the lens.

It is still not clear how the condition of pseudoexfoliation (PXF) of the lens capsule develops. The material appears to be an amyloid-like substance with fibrils embedded in a ground substance, which appears at a variety of ocular and extraocular sites. ${ }^{1-11}$ The vascular changes described by Vannas ${ }^{12}{ }^{13}$ are very marked, and their relationship to the pseudoexfoliative process is of particular interest.

Accordingly we have re-examined these vascular changes and also measured the leakage of fluorescein in a group of patients in order to determine whether there is any evidence of (1) vascular changes or increased fluorescein leakage occurring in the clinically unaffected fellow eye in patients with unilateral PXF; (2) increased fluorescein leakage in the posterior segment of affected eyes, with heavy leakage in the anterior segment; (3) change in the pattern of the vascular reaction and in the fluorescein leakage following cataract extraction in affected eyes.

Correspondence to W. E. Gillies, 110 Collins Street, Melbourne, Victoria 3000, Australia.

\section{Materials and methods}

Fluorescein angiography was performed on 17 patients with bilateral PXE, with fluorophotometry in 9 of these, and on 14 patients with clinical unilateral PXF, 11 of these having fluorophotometry. Angiography and fluorophotometry were also performed on 12 bilateral aphakes (10 of these had bilateral PXF, in 2 it was unilateral) and 7 unilateral aphakes with bilateral PXF. In addition 25 patients of comparable age without PXF, 11 aphakic (6 with bilateral aphakia), were studied.

Iris angiography was performed with a Nikon camera and 105 Macro lens attached to the side of the Zeiss fundus angiography camera, utilising its light source and filters; this left the eyepiece of the angiogram camera free for use by an observer. For angiography the subjects received an intravenous bolus of $14 \mathrm{mg} / \mathrm{kg}$ body weight $25 \%$ sodium fluorescein. At the conclusion of photography the patient's pupils were dilated with cyclopentolate hydrochloride $1 \%$, and when necessary phenyl- 


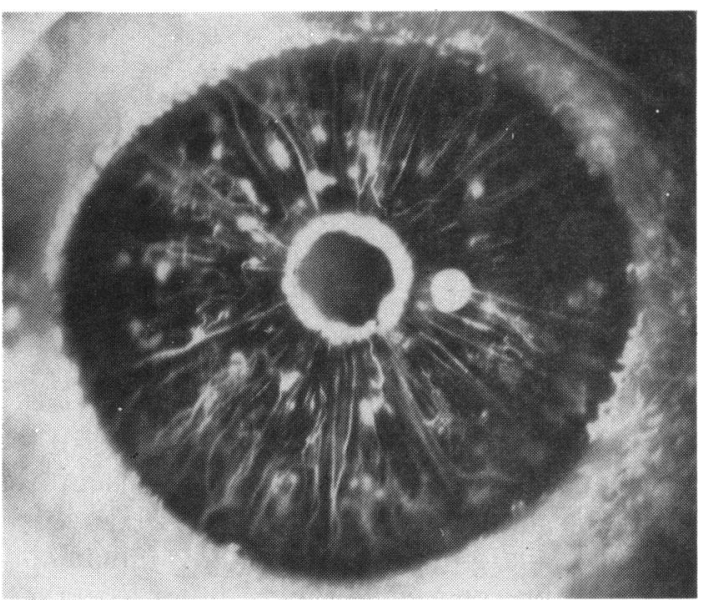

Fig. 1 Male aged 62 years with bilateral PXF. Fluorescein angiogram at 42 seconds shows heavy pupillary leak of dye, neovascular tufts, some loops and more complex neovascular patterns, with some areas ofloss of radial vessels.

ephrine hydrochloride $10 \%$ was also used. Fluorophotometry readings were taken one hour after fluorescein injection by means of the Metricon model 120 slit-lamp fluorophotometer after the application of a Goldmann contact lens. Fluorescein concentrations were measured at both an anterior focus with the focal plane at the level of the posterior lens capsule and posteriorly where the average of 6 points lying from 3 to $5 \mathrm{~mm}$ anterior to the chorioretinal peak was taken. The technique is described in detail elsewhere. ${ }^{14}$

Statistical analysis of the data was by the Mann-

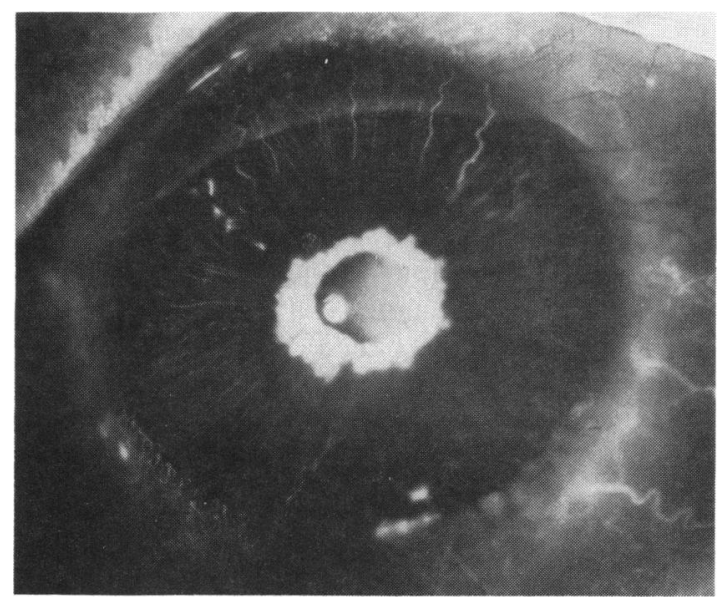

Fig. 2 Female aged 82 years with bilateral PXF. Iris angiogram at 30 seconds shows complete neovascularisation of the inner $1 / 3$ of the iris, with heavy leak of dye.
Whitney test unless otherwise stated. Data are expressed as mean \pm standard deviation.

\section{Results}

BILATERAL PSEUDOEXFOLIATION

These patients showed extensive vascular changes which may be summarised as follows.

(a) Loss of radial vessels with frequent sector filling defects, both arteries and veins being affected (Fig. 1).

(b) Leak of fluorescein from the pupil margin. This seemed to be a universal sign in pseudoexfoliation and was much heavier than the small leak which often occurs in patients of this age group. It seemed to come from neovascular tufts at the pupil margin which were not apparent on slit-lamp examination (Figs. 1 and 4A).

(c) Diffuse neovascularisation of the iris stroma, which showed fluorescein leakage (Figs. 1,2). This appeared as (i) small neovascular tufts; (ii) neovascular loops which were large and ran radially or at times circumferentially; (iii) in some patients more extensive and complex patches of neovascularisation, the impression being of a progressive process from simple to complex patterns secondary to the loss of radial vessels; $(d)$ neovascularisation of the inner $1 / 3$ of the iris. In some patients there was an extensive neovascular network of capillaries which was either patchy or complex and which leaked fluorescein. This was a less common finding than the preceding ones, but was usually present to some degree (Figs. 2, 3A).

The fluorophotometry results at the anterior focus are shown in Table 1 . The patients were subdivided on the basis of iris colour into those with blue and those with nonblue irides, as the iris pigmentation has been shown to influence the fluorescence at this focus. ${ }^{14}$ In all but 3 of the 17 affected eyes the fluorescein concentration at the anterior focus was higher than the normal mean, which is highly significant $(\mathrm{p}<0.001$ by the binomial theorem); in 9 of these it was higher than the estimated control normal range (mean \pm 2 standard deviations), which is highly significant $(p<0 \cdot 001)$. Overall, the fluorescein con-

Table 1 Anterior fluorescence in phakic subjects: comparison of normal control subjects with patients with bilateral pseudoexfoliation

\begin{tabular}{cll}
\hline $\begin{array}{l}\text { Fluorescein } \\
\text { concentration } n g / m l\end{array}$ & $\begin{array}{l}\text { Normal } \\
\text { controls }\end{array}$ & $\begin{array}{l}\text { Bilateral } \\
\text { pseudoexfoliation }\end{array}$ \\
\hline Iris colour: blue & $438 \cdot 2 \pm 58 \cdot 0$ & $603 \cdot 2 \pm 206 \cdot 0$ \\
nonblue & $301 \cdot 5 \pm 68 \cdot 6$ & $608 \cdot 2 \pm 226 \cdot 1$ \\
total & $368 \cdot 8 \pm 94 \cdot 7$ & $606 \cdot 0 \pm 210 \cdot 3$ \\
\hline
\end{tabular}

All values mean \pm standard deviation.

*Pseudoexfoliation compared with normal controls significant to $\mathrm{p}<0 \cdot 01$. 


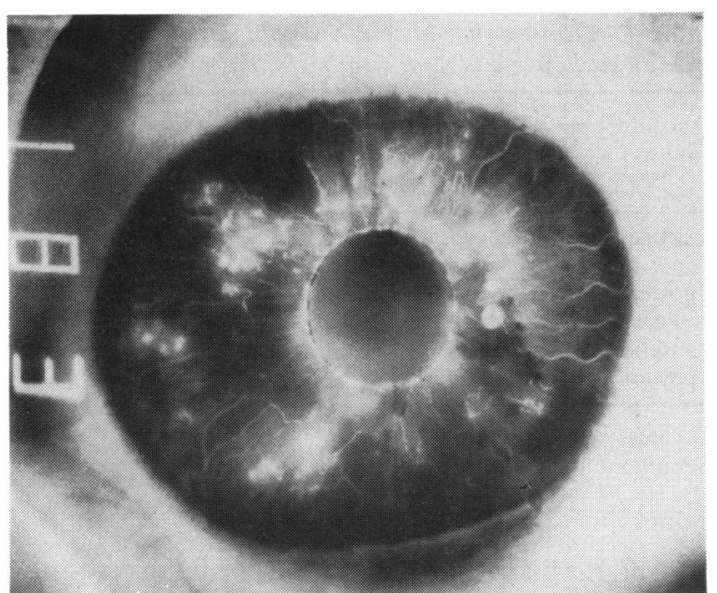

Fig. 3A

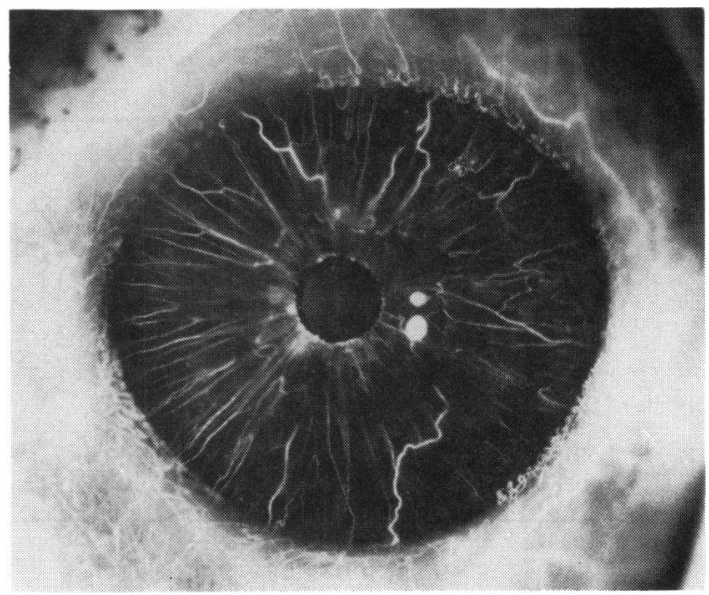

Fig. 3B

Fig. 3A Female aged 64 years with unilateral PXF. Iris angiogram at 37 seconds shows marked loss of radial vessels and extensive neovascularisation. B Left unaffected eye at 37 seconds shows some very early neovascular changes with leakage which may be seen in a normal patient in this age group.

centration at the anterior focus was significantly higher in the patients with bilateral PXF than in the normal controls $(p<0 \cdot 01)$.

At the posterior focus the fluorescein concentration was within the estimated normal range in 10 of the 14 eyes with bilateral PXF, but this was above the normal range in 4 ; this increase is significant $(p<0 \cdot 001$, binomial theorem). As a group the posterior fluorescein concentration was $14 \cdot 2 \pm 2 \cdot 8 \mathrm{ng} / \mathrm{ml}$, which is significantly higher than that of the normal controls of $10 \cdot 1 \pm 2 \cdot 8 \mathrm{ng} / \mathrm{ml}(\mathrm{p}<0 \cdot 01)$.

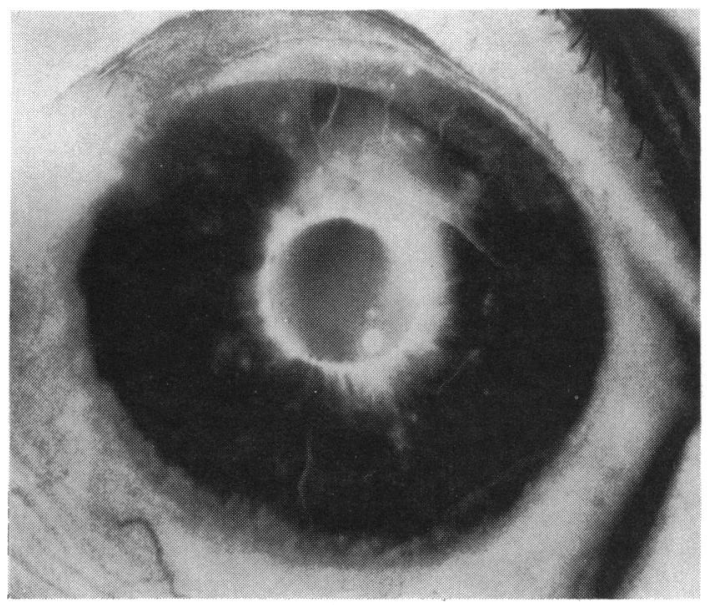

Fig. 4A
UNILATERAL PSEUDOEXFOLIATION

On iris angiography the eyes affected by pseudoexfoliation showed neovascular changes similar to those already noted for patients with bilateral PXF. The unaffected eyes showed much less vascular change in all cases, though all cases showed some pupillary leak, which was not heavy in any case. Some eyes showed leaking points in the stroma, and some showed sector filling defects in the iris. These changes were no more than might be observed in some normal eyes in a similar age group but were more common in

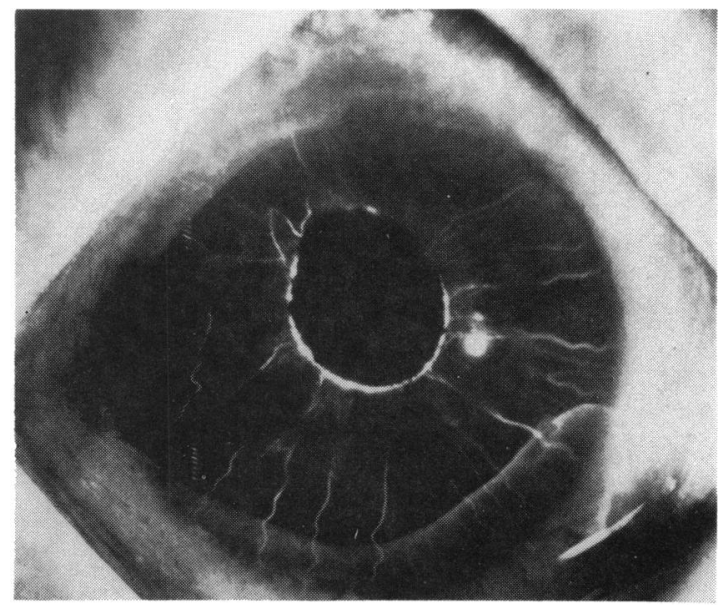

Fig. 4B

Fig. 4A Female aged 76 years with bilateral $P X F$ and unilateral aphakia. Phakic eye shows very heavy leak of dye at 46 seconds causing blurring of the picture. B Aphakic fellow eye 7 years postoperatively, originally with the same degree of $P X F$ as the other eye, now shows only slight pupillary leak of dye at 44 seconds. 
Table 2 Anterior fluorescence in phakic subjects with unilateral pseudoexfoliation: comparison of the affected eye with the unaffected fellow eye

\begin{tabular}{lll}
\hline $\begin{array}{l}\text { Fluorescein } \\
\text { concentration } n g / m l\end{array}$ & Affected eye & Unaffected fellow eye \\
\hline Blue irides & $571 \cdot 6$ & $312 \cdot 6$ \\
& $527 \cdot 0$ & $316 \cdot 1$ \\
& $895 \cdot 1$ & $367 \cdot 3$ \\
& $477 \cdot 2$ & $448 \cdot 0$ \\
& $820 \cdot 8$ & $536 \cdot 9$ \\
Nonblue irides & $619 \cdot 3$ & $556 \cdot 9$ \\
& $448 \cdot 7$ & $296 \cdot 2$ \\
& $419 \cdot 7$ & $399 \cdot 5$ \\
& $513 \cdot 6$ & $424 \cdot 1$ \\
& $1040 \cdot 2$ & $895 \cdot 4$ \\
\hline
\end{tabular}

Table 3 Anterior fluorescence in patients with bilateral pseudoexfoliation: comparison of phakic and aphakic eyes

\begin{tabular}{lll}
\hline Range & \multicolumn{2}{l}{ Number of patients } \\
\cline { 2 - 3 } & Phakic & Aphakic \\
\hline Mean -2 SD)* to mean $_{\text {Mean to (mean+2SD) }}$ & 3 & 3 \\
Greater than 2 SD above mean & 5 & 7 \\
\hline
\end{tabular}

${ }^{*} \mathrm{SD}=$ standard deviation.

that most eyes showed some evidence of pupillary leak or early neovascularisation (Figs. 3 A,B).

In all 10 cases the fluorescein concentration at the anterior focus in the affected eye was higher than that in the unaffected fellow eye (Table 2$)(p<0.001$, standard rank test).

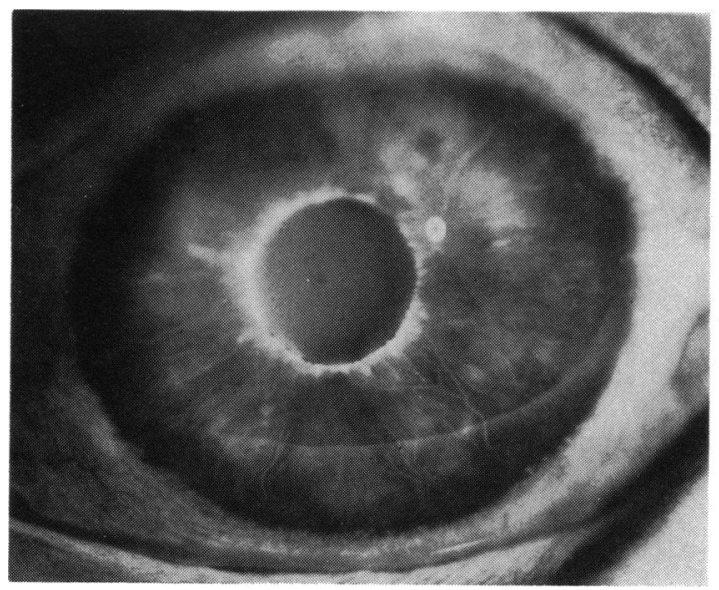

Fig. 5A
Table 4 Fluorescence in aphakes: comparison of pseudoexfoliative and nonpseudoexfoliative (normal) eyes

\begin{tabular}{lcc}
\hline \multirow{2}{*}{$\begin{array}{l}\text { Time since operation, } \\
\text { months }\end{array}$} & \multicolumn{2}{c}{ Fluorescein concentration, $\mathrm{ng} / \mathrm{ml}$} \\
\cline { 2 - 3 } & Normal & Pseudoexfoliation \\
\hline Less than 12 months: & $628 \cdot 5 \pm 310 \cdot 6$ & $889 \cdot 5 \pm 525 \cdot 2$ \\
$\quad$ anterior & $39 \cdot 0 \pm 20 \cdot 8$ & $35 \cdot 3 \pm 23 \cdot 9$ \\
posterior & & \\
Greater than 12 months: & $315 \cdot 8 \pm 120 \cdot 8$ & $685 \cdot 1 \pm 352 \cdot 0^{*}$ \\
$\quad$ anterior & $46 \cdot 9 \pm 16 \cdot 7$ & $35 \cdot 6 \pm 26 \cdot 1$ \\
posterior &
\end{tabular}

All values mean \pm standard deviation.

*Significant at $\mathrm{p}<0.01$ level compared with normal.

In all, the fluorescein concentration at the posterior focus was higher in the eye with PXF than in the unaffected fellow eye $(p<0 \cdot 02$, standard rank test), and the concentration in the unaffected fellow eye did not differ significantly from that of the normal controls.

\section{BILATERAL APHAKIA}

The iris angiograms showed a very marked loss of radial markings, and there was considerable neovascularisation. Most patients, however, showed much less pupillary leak of fluorescein when compared with pseudoexfoliative eyes not operated upon (Figs. 4B, 5B).

The ranges of fluorescein concentrations at the anterior focus in both phakic and aphakic patients with bilateral PXF are shown in Table 3. There is no significant difference between the 2 groups.

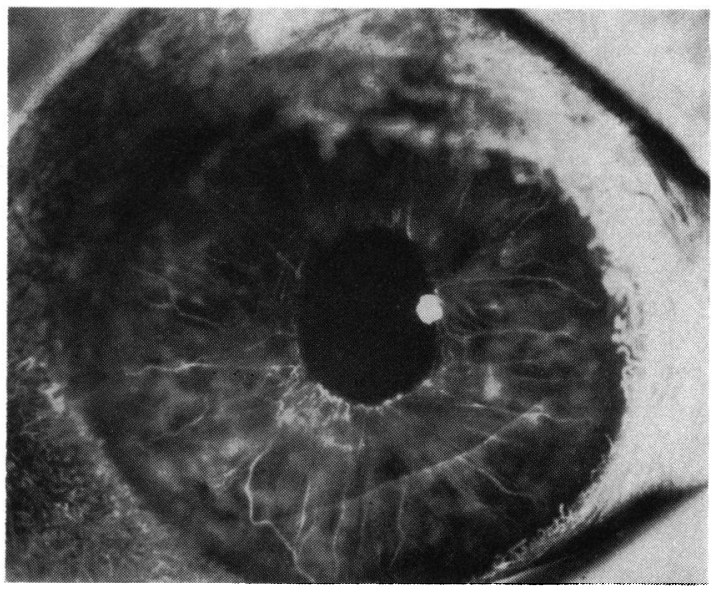

Fig. 5B

Fig. 5A Male aged 74 years with bilateral PXF. Preoperative iris angiogram at 55 seconds shows iris neovascularisation and pupillary leak of dye causing some blurring of the picture. B The same eye as in Fig. 5 A 11 months postoperatively. Iris angiogram at 55 seconds shows marked diminution in the pupillary leak of dye. 
UNILATERAL APHAKIA WITH BILATERAL PSEUDOEXFOLIATION

On iris angiography the diminution of pupillary leakage of dye in the aphakic eye compared with the phakic fellow eye was striking (Figs. 4A,B), and in one patient with preoperative and postoperative angiograms this diminution is well shown (Figs. $5 A, B)$. In all cases the fluorescein concentration at the anterior focus of the aphakic eye was within the upper normal range and lower than that of the fellow phakic eye $(p<0.02$, standard rank test). Of the fellow phakic eyes all but one were above the normal range.

In aphakes with PXF less than 12 months postoperatively the fluorescein concentration at the anterior focus did not differ significantly from normal aphakic eyes (Table 4). With time the fluorescein concentration fell, and this was more marked in the normal subjects, so that at more than 12 months postoperatively the anterior fluorescein concentration was significantly higher in the patients with PXF than in the normal subjects, though in 11 of these 21 aphakic eyes with PXF the anterior level had returned to within the normal range. The posterior vitreous fluorescein concentration in aphakes with PXF did not differ significantly from that of the normal aphakes of similar time since operation.

\section{Comment}

We confirmed the neovascular changes affecting the iris with partial closing off of the normal radial iris vasculature, the development of neovascularisation of increasing complexity in the outer $2 / 3$ of the iris stroma, development of a fine leaking capillary network in the inner $1 / 3$ of the iris stroma in some cases, and heavy fluorescein leakage from the pupil margin in all cases.

Fluorophotometry measures the heavy leakage of fluorescein from the anterior segment of the eye, but this is not associated with a corresponding increase in fluorescein leakage from the posterior segment in PXF (correlation coefficient 0.4 ), unlike the rise seen in diabetics with rubeosis iridis. The vascular reaction which occurs in PXF seems to be located predominantly in the anterior segment of the eye, suggesting that the process of production of PXF is primarily in this area.

Consideration of unilateral cases of PXF shows that, although there is evidence of very early vascular changes in unaffected eyes, this does not lie outside normal limits. Although the early development of pseudoexfoliative material may precede its clinical detection, the vascular reaction does not, so that it seems to be either a secondary process or else part of a more complex process which produces the material.
The leak of fluorescein appears to be associated with the vascular disorder and does not precede it.

In aphakic pseudoexfoliative eyes, iris angiography indicated a very marked loss of radial vessels which may be due to the disease process or may be exacerbated by cataract extraction. The striking diminution in pupillary leak in aphakic cases may be a mechanical effect from operation, but it persists after 12 months.

Fluorophotometry supports this decrease in leakage with time after cataract extraction, and, although the anterior fluorescein concentration in the aphakic eyes with PXF reaches a plateau at a higher level than that found in normal aphakes, it is lower in the aphakic than phakic eye of patients with bilateral PXF, suggesting that the progression of the neovascular process is halted or slowed after cataract extraction.

Pseudoexfoliative material may be produced in the anterior segment of the eye as a reaction to the presence of an aging lens, either from the lens itself or from other sites, or it may be produced quite independently of the presence of the lens. It has been claimed that removal of the lens causes some regression of the process, ${ }^{15} 16$ though this has been contested. ${ }^{17} 18$ Pseudoexfoliative material is present in considerable quantity, and much of it will be left after lens extraction; further it is very resistant to enzyme action, therefore it will persist long after cataract extraction. The present investigations suggest some regression of the vascular reaction, which is consistent with a regression in production of pseudoexfoliative material.

We would emphasise the extensive neovascular component of the pseudoexfoliative process and that this is probably secondary in nature. The neovascular process probably does not continue to develop after lens extraction, suggesting that the presence of the lens is important to the process.

We thank Glenys van den Brenk for her skill in the photographic work. Judith Quilter for her help with references, Kerrie Port for secretarial assistance, and the Melbourne University Department of Ophthalmology for its help.

This project was carried out under research projects 20 and 55 of the RVEEH.

\section{References}

1 Bertelsen TI. Fibrillopathia epithelio-capsularis. The so-called senile exfoliation or pseudo-exfoliation of the anterior lens capsule. Acta Ophthalmol (Kbh) 1966; 44: 737-50.

2 Ringvold A. A preliminary report on the amino acid composition of the pseudo-exfoliation material (PE material). Exp Eye Res 1973; 15: 37-42.

3 Ringvold A. On the occurrence of pseudo-exfoliation material in the extrabulbar tissue from patients with pseudo-exfoliation syndiome of the eye. Acta Ophthalmol (Kbh) 1973; 51: 411-8. 
4 Ringvold A. Husby G. Pseudo-exfoliation material-an amvloid-like substance. Exp Eve Res 1973; 17: 289-99.

5 Davanger $\mathrm{M}$. On the molecular composition and physico chemical properties of the pseudo-exfoliation material. Acta Ophthalmol (Kbh) 1977: 55: 621-33.

6 Meretoja J. Tarkkanen A. Occurrence of amyloid in eyes with pseudo-exfoliation. Ophthalmic Res 1977; 9: 80-91.

7 Davanger M. Hovig T. Pseudo-exfoliation fibrils examined by negative staining. Acta Ophthalmol (Kbh) 1978: 56: 226-32.

8 Davanger $M$. On the interfibrillar matrix of the pseudoexfoliation material. Acta Ophthalmol (Kbh) 1978; 56: 233-40).

9 Dickson DH. Ramsav MS. Fibrillopathia epitheliocapsularis. Review of the nature and origin of pseudoexfoliative deposits. Trans Ophthalmol Soc UK 1979: 99: 284-92.

10) Davanger M. On the ultrastructure and the formation of pseudoexfoliation material. Acta Ophthalmol (Kbh) 1980; 58: 520-7.

11 Davanger M. Pedersen OO. Pseudo-exfoliation material on the anterior lens surface. Demonstration and examination of an interfibrillar ground substance. Acta Ophthalmol (Kbh) 1975: 53: $3-18$.
12 Vannas A. Fluorescein angiography of the vessels of the iris in pseudoexfoliation of the lens capsule. capsular glaucoma and some other forms of glaucoma. Acta Ophthalmol (Kbh) 1969; 105: (suppl) 9-75.

13 Vannas A. Vascular changes in pseudoexfoliation of the lens capsule and capsular glaucoma. A fluorescein angiographic and electron microscopic study. Albrecht von Graefes Arch Klin Ophthalmol 1972; 184: 248-53.

14 Brooks AMV. Experiences with fluorophotometry. Aust $J$ Ophthalmol 1982; 10: 41-51.

15 Gillies WE. The effect of lens extraction in pseudoexfoliation of the lens capsule. Br J Ophthalmol 1973; 57: 46-51.

16 Gillies WE. Secondary glaucoma associated with pseudoexfoliation of the lens capsule. Trans Ophthalmol Soc UK 1978; 98: $96-100$.

17 Sugar HS. Harding C. Bursky D. The exfoliation syndrome. Ann Ophthalmol 1976; 18: 1165-81.

18 Sugar HS. The exfoliation syndrome: source of the fibrillar material on the capsule. Surv Ophthalmol 1976; 21: 59-64. 